\title{
Análisis del deporte femenino español de competición desde la perspectiva deprotagonistas clave Analysis of Spanish women's sport competition from the perspective of key players
}

\author{
Ma Teresa Leruite Cabrera, Pilar Martos Fernández, Mikel Zabala Díaz \\ Universidad de Granada (España)
}

\begin{abstract}
Resumen. El progreso de las deportistas en términos de igualdad en la sociedad española no sólo se mide por el acceso de más mujeres al deporte o a la toma de decisiones sino que es importante conocer aspectos como su realidad social y las dificultades con las que se encuentran. En el presente artículo se analiza el estado de la cuestión del deporte femenino español de competición desde una perspectiva de género teniendo en cuenta los siguientes factores: el marco normativo en igualdad de trato y sin discriminación en el deporte, las políticas deportivas con perspectiva de género llevadas a cabo por el Consejo Superior de Deportes y las federaciones deportivas, la discriminación por razón de género, los medios de comunicación social y el lenguaje sexista. El estudio se posiciona en el paradigma interpretativo, utilizando la metodología cualitativa. Se recoge la opinión de 9 entrevistados especializados en la materia: 3 gestores autonómicos, 2 gestoras del ámbito federativo, 1 jurista deportiva, 1 periodista deportiva y 2 ex-profesoras universitarias e investigadoras expertas en género y deporte. El instrumento utilizado ha sido la entrevista semiestructurada. Los resultados obtenidos revelan un panorama necesario de cambio, donde convendría una aplicación exhaustiva del marco normativo con perspectiva de género al ámbito deportivo, la revisión de las políticas y programas con perspectiva de género, llevadas a cabo por las federaciones deportivas y una modernización de la gestión federativa.
\end{abstract}

Palabras clave. Federaciones deportivas españolas, perspectiva de género, deportistas españolas, cultura de las organizaciones, alta competición.

\begin{abstract}
The progress of athletes in terms of equality in the Spanish society is not only measured by the access of women to sport or decision-making, but also by knowing aspects such as social reality and the difficulties they face. In this article the state of the knowledge of Spanish women's sport competition is analyzed from a gender perspective taking into account the following factors: the regulatory framework on equality and no discrimination in sport, sport policies with a gender perspective carried out by the Consejo Superior de Deportes and sport federations, gender discrimination, mass media and sexist language. The study stands in an interpretive paradigm and uses a qualitative methodology. Nine expert interviews are collected: three autonomous managers, two female federative managers, one sports lawyer, one sports journalist and two former university professor experts in gender and sport. The instrument used was the semi-structured interview. The results reveal a need for changing the reality. There is a need for a comprehensive implementation of the regulatory framework with a gender perspective to the sports field, the review of policies and programs with a gender perspective carried out by the sports federations and a modernization of federation management.
\end{abstract}

Keywords: Spanish sport federations, gender perspective, Spanish female athletes, culture of organizations, high performance.

\section{Introducción}

El progreso de las deportistas en términos de igualdad en la sociedad española no sólo se mide por el acceso de más mujeres al deporte o a la toma de decisiones sino que es importante conocer aspectos como su realidad social y las dificultades con las que se encuentran (Alfaro, Vázquez, Gallardo \& Ferro, 2012; González, 2010; Torres, 2005).

Sin embargo, y al amparo del marco teórico que ofrece la Teoría de Sistemas (Bertalanffy, 1968), en la actualidad la deportista no puede ser entendida sin una concepción de ella misma y su entorno.

Así, elementos como la propia deportista y su entorno próximo, los medios de comunicación social, el marco normativo en igualdad de trato y sin discriminación en el deporte, las instituciones deportivas y el acceso de las mujeres a los puestos de dirección en las organizaciones deportivas conforman entre otros una extensa y compleja red de relaciones intrínsecas y extrínsecas susceptibles de configurar los sistemas sociales. La desigualdad entre hombres y mujeres es sin duda una lacra social, no sólo las cifras referentes a la violencia de género son indicativas, como muestran las muertes por violencia de género que ascienden a 41 este año o más concretamente a los casos de abuso y acoso sexual en el deporte en diferentes países (Brackenridge,2001; Svela Sand, Fasting, Chroni \& Krorre, 2011; Vázquez, Bastanchuri \& Fernández, 2002) sino también a aquellas derivadas del trato desigual en otros ámbitos, por ejemplo, respecto a la brecha salarial entre hombres y mujeres; de un 16,4\% en España, y a la desigualdad en los roles asociados a la deportista en diversos planos (el trabajo, la publicidad, el lenguaje sexista).Asimismo, los medios de comunicación han contribuido a perpetuar la dominación masculina existiendo una exclusión de las mujeres en las retransmisiones deportivas, siendo los eventos deportivos más retransmitidos aquellos que refuerzan los estereotipos sociales de género (Alfaro et al., 2012; Bissell \& Duke, 2007).

En referencia a la participación de la deportista española en el

Fecha recepción: 10-04-14- Fecha envío revisores: 13-04-14- Fecha de aceptación: 06-12-14 $\mathrm{M}^{\mathrm{a}}$ Teresa Leruite Cabrera mayteleruite@ugr.es deporte federado español los estudios científicos de los últimos años revelan que el número de mujeres que participan en el deporte de competición sigue siendo muy inferior al de los hombres (Alfaro et al., 2012; Puig \& Soler, 2003,2004; Robles \& Escobar, 2007). En total de las 66 federaciones nacionales existentes, 712.027 (20,9\%) son licencias federativas femeninas y 2.682.608 (79\%) son masculinas (Consejo Superior de Deportes, 2013). La menor presencia de las mujeres españolas en el mundo del deporte de competición se ha reflejado a lo largo de su historia.

Haciendo un breve repaso histórico sobre la participación femenina española observamos que en los primeros sesenta años de existencia de los juegos olímpicos de la era moderna es prácticamente nula a excepción de mujeres que compitieron en los Juegos de París de 1924 y de Roma de 1960. El estallido de la Guerra Civil Española y el triunfo del Franquismo sobre la II República Española supuso el retroceso durante 3 décadas del ideal progresista y modernizador del deporte femenino impulsado por el gobierno republicano hacia una concepción deportiva basada en la moral católica conservadora del nuevo régimen franquista (García Ferrando, 1987; Manrique, 2003; Manrique, López, Torrega, \& Mangos, 2008).

Es a partir de 1975 en la transición demográfica cuando junto con la incorporación de la mujer a ámbitos sociales como el trabajo, la educación y la política y la nueva Ley de 13/1980, de 31 de marzo, General de la Cultura Física y del Deporte, de 1980 que propicia el debate social sobre el rol de la mujer en el deporte, los nuevos programas de fomento de la actividad física a todos los niveles y la dotación de instalaciones públicas deportivas por todo el territorio nacional se incrementa la participación de las mujeres en todas las esferas del deporte y en especial en el deporte de alta competición.

A partir de los Juegos Olímpicos de los Ángeles de 1984 se observa el incremento paulatino de la participación femenina española, siendo en los Juegos Olímpicos de Barcelona de 1992 donde la mujer española se consolida en el olimpismo, con 141 mujeres que representaron el 29,7\% de los atletas españoles, consiguiendo las primeras medallas de oro femeninas (hockey, judo, vela y gimnasia) para el deporte español y un $11^{\circ}$ puesto en el medallero femenino de potencias mundiales. La 
mujer española en la alta competición, aunque tardía ha cosechado multitud de éxitos. Desde su primera participación en París de 1924 hasta la actualidad el crecimiento ha sido muy acelerado llegando a un 43\% de participación femenina española en los Juegos Olímpicos de Pekín y consagrándose con un total de 135 mujeres deportistas en los Juegos Olímpicos de Londres, superando por primera vez a los deportistas españoles en el medallero consiguiendo 11 medallas para la delegación española, siendo bautizados en España los Juegos Olímpicos de Londres como los «Juegos de las Mujeres».

Por otra parte, en una recopilación de artículos sobre los principales puestos en las organizaciones deportivas de varios países las mujeres están infrarrepresentadas en todas las áreas y niveles (Alfaro et al., 2012; Hartmann-Tens \& Pfister, 2003; Pfister, 2010). Las mujeres han disminuido su presencia tanto en las Asambleas Generales de las Federaciones como en las Juntas Directivas de las Federaciones alcanzando sólo la cuota del 20\% en los Comités de Árbitros y Jueces (ver tabla 1).

Tabla 1.

Miembros en órganos de gobierno de las federaciones deportivas españolas (Adaptado del CSD 2013) Miembros en órganos de gobierno 2013

\begin{tabular}{lcccc} 
& Hombres & Mujeres & Total es & $\%$ Mujeres \\
Asambleas generales & 4055 & 501 & 4556 & $11 \%$ \\
Comisiones delegadas & 642 & 76 & 718 & $10,6 \%$ \\
Juntas directivas & 801 & 112 & 913 & $12,3 \%$ \\
Comités técnicos de árbitros y jueces & 1130 & 358 & 1488 & $24 \%$ \\
Miembros en otros comités & 1690 & 341 & 2031 & $16,8 \%$ \\
TOTAL ÓR GANOS DE GOBIER NO & 8318 & 1388 & 9706 & $14,3 \%$ \\
\hline
\end{tabular}

En el Consejo Superior de Deportes (CSD) actualmente de 14 miembros 5 son mujeres, representando el 35,71\%, existiendo sólo una mujer que haya desempeñado la Presidencia del CSD a lo largo de la historia de este organismo. En el Comité Olímpico Español (COE) las mujeres no alcanzan la cuota del $20 \%$ de representación y sólo existe una mujer directiva que desempeña actualmente la vicepresidencia del COE desde hace 10 años. Así mismo, sólo hay tres presidentas de las 66 federaciones deportivas españolas (deportes de hielo, petanca y salvamento y socorrismo) que siguen desempeñando su cargo desde 2002.

A raíz de estas desigualdades los gobiernos han ido implementando marcos políticos normativos en materia deportiva para reducir la brecha de desigualdad existente de la deportista en todos los ámbitos de participación. La legislación deportiva en materia de igualdad de género en el deporte a nivel español no existe como tal y se encuentra integrada en diversas políticas de igualdad llevadas a cabo por el gobierno, instituciones y organismos autonómicos (Robles \& Escobar, 2007). La Ley Orgánica 3/2007, de 22 de marzo, para la igualdad efectiva de mujeres y hombres recoge en su artículo 29 una mención al deporte instando a promover el deporte femenino por parte del gobierno español, garantizando la igualdad real y efectiva entre mujeres y hombres y favoreciendo la efectiva apertura en las disciplinas deportivas a las mujeres, mediante el desarrollo de programas específicos en todas las etapas de la vida y en todos los niveles, incluidos los de responsabilidad y decisión. Al amparo de la Orden Pre/525/2005, de 7 de Marzo, se crea por primera vez una Unidad con carácter permanente para desarrollar el programa de «Mujer y Deporte». Desde que en 2007 el Consejo Superior de deportes concediera ayudas a los programas de Mujer y Deporte de 17 federaciones españolas, en 2013 han sido 47 las federaciones subvencionadas, suponiendo un incremento del $74 \%$.

Una vez conocidas las bases del contexto sociocultural de la mujer en el deporte de competición a nivel español, los objetivos del estudio se centran en analizar la situación de la deportista española en el deporte de alta competición a través del discurso de protagonistas clave atendiendo para ello a elementos que influyen en el microsistema deportivo de las deportistas tales como: el marco normativo en igualdad de trato y sin discriminación en el deporte, las políticas deportivas con perspectiva de género llevadas a cabo por el Consejo Superior de Deportes y las federaciones deportivas, la discriminación por razón de género y violencia machista y los medios de comunicación social y lenguaje sexista.

\section{Metodología}

\section{Método}

El desarrollo de esta investigación se posiciona en el paradigma interpretativo utilizando una metodología cualitativa con un carácter descriptivo, relacional e interpretativo (Fraile \& Vizcarra, 2009).

\section{Participantes}

La población del universo de estudio está formada por nueve personajes clave relacionados con la perspectiva de género en España en los diversos sectores del ámbito deportivo en los que desarrollan su actividad profesional: 3 gestores deportivos de las Comunidades Autónomas relacionados con las gestión federativa (sujetos 1, 2 y 3), 2 gestoras del ámbito federativo de nivel nacional e internacional (sujetos 4 y 5), 1 abogada especializada en derecho deportivo (sujeto 6), 1 periodista experta en género y deporte (sujeto 7) y 2 ex-profesoras universitarias e investigadoras sobre la temática de mujer y deporte de reconocido nivel internacional (sujeto 8 y 9). Para la selección de las y los protagonistas se tuvo en cuenta su perfil profesional y su reconocido prestigio a nivel nacional y en algunos casos a nivel internacional. Además, siguiendo el objetivo del estudio se pretende analizar la situación de la deportista desde todos los sectores que engloban el sistema deportivo español de competición.

\section{Instrumento}

Se ha decidido utilizar como instrumento la entrevista semiestructurada. Se decidió diseñar las dimensiones y los items a través del método Delphi, cuyo proceso garantiza la validez del contenido (Landerta, 1999).Se seleccionaron 3 profesores universitarios, (1 profesor y 2 profesoras) expertos en la temática y conocedores del procedimiento. Se comenzó informando sobre la temática: percepción y conocimiento que tienen las personas expertas a entrevistar sobre la situación de la deportista española de competición en España y se realizó la siguiente pregunta ¿qué grandes dimensiones habría que abordar para elaborar una entrevista de esta temática?. Una vez analizadas las dimensiones por el grupo de profesores se procedió a realizar las preguntas de las entrevistas por un comité de dos expertos siguiendo las recomendaciones propuestas de autores como Bryman, (2004); Fink, (1995). Para captar la información necesaria para los objetivos del estudio, se decidió partir de algunas de las variables del cuestionario del estudio «Análisis del modelo del deporte español del siglo XIX» (Cabello, Rivera, Trigueros \& Pérez, 2011) y del estudio sobre las políticas de género en los gobiernos nacionales ingleses de (Shaw \& Penney, 2003). Además, se utilizaron como documentos de apoyo para la construcción del sistema de categorías los siguientes: guión de entrevista previo a los participantes, análisis de fuentes documentales tales como reglamentos y estatutos federativos, marcos normativos a nivel internacional y nacional sobre perspectiva de género, estadísticas del Consejo Superior de Deportes y estudios científicos previos relacionados con la temática de mujer y deporte y objetivos planteados en la investigación. Finalmente se realizó una entrevista piloto antes de la realización definitiva de las entrevistas a los participantes. Una vez validadas las entrevistas y una vez ajustadas, se dieron por válidas para su utilización. Las entrevistas estaban estructuradas finalmente por 4 dimensiones y 16 preguntas.

\section{Entrevista}

1. Aspectos de política deportiva y marco normativo

-¿Cómo considera la situación actual de la mujer española en el deporte de competición? ¿Y con respecto a otros países?

-En líneas generales ¿Cómo considera la actual Ley del deporte de 1990 en referencia al deporte femenino? ¿Deberían de tomarse medidas adicionales?

- ¿Considera que es necesaria la profesionalización del deporte femenino para igualar en condiciones a los hombres? ¿Cómo puede sustentarse en algunos deportes más minoritarios? 
-¿Qué balance puede hacerme de la reciente aprobada ley de Igualdad de 1997? ¿Se ha mejorado el deporte femenino? ¿Son necesarias más actuaciones?

-¿Cómo considera que se está trabajando desde las federaciones y demás organismos deportivos respecto a la igualdad entre mujeres y hombres?

-¿Considera importante la presencia de las mujeres en los órganos directivos de las organizaciones deportivas para mejorar el deporte practicado por las deportistas?

-¿Considera que el personal directivo actual de las organizaciones deportivas tiene formación y visión relacionada con la perspectiva de género?

-¿Considera necesario que se creen más órganos específicos con perspectiva de género como la «Comisión de Mujer y Deporte» en las federaciones y demás organismos deportivos en los que la mujer está infrarrepresentada? ¿Qué características y funciones deberían cumplir?

2. Aspectos de discriminación sexual/acoso sexual

- ¿Considera que las deportistas en los inicios y a lo largo de su carrera profesional tienen que hacer frente a alguna/s barrera/as que por el hecho de ser mujer dificultan el desarrollo de su actividad deportiva?

- ¿Tienen que demostrar mayor nivel de exigencia para llegar al mismo reconocimiento que los hombres?

-¿En qué medida cree que está reconocida la labor de las deportistas en la sociedad? (nada, muy poco, poco, suficiente, bastante, mucho)?

-Estudios científicos recientes a nivel internacional en mujeres deportistas muestran la violencia y el acoso sexual como uno de los problemas que están sufriendo las deportistas a todos los niveles de práctica deportiva ¿Cree que se está abordando este asunto por parte de los organismos deportivos?

3. Aspectos socioeconómicos

- ¿Estima que los ingresos derivados de becas, premios percibidos por las deportistas son iguales a los percibidos por los deportistas en idéntico nivel o situación profesional?

4. Medios de comunicación y lenguaje sexista

-¿Qué opina del lenguaje sexista en el deporte? ¿Qué supondría la implantación de un lenguaje más inclusivo, mejoraría la situación de la mujer en el deporte?

-¿Qué opina de la presencia y el trato que reciben las deportistas en los medios de comunicación?

-Actualmente, ¿Cómo considera la investigación relacionada con la mujer y el deporte? ¿Sobre quéáreas sería necesario investigarse?

\section{Procedimiento y análisis de datos}

Toda la fase del procedimiento de recogida de información (localización, identificación, contacto de las personas, realización de la entrevista, registro de información, y la recogida de información obtenida) fue realizada por un único investigador con la finalidad de obtener una mayor fiabilidad y validez del cuestionario (García Ferrando, 2002). Siete fueron entrevistados mediante videoconferencia y dos telefónicamente. Una grabadora fue utilizada durante las entrevistas tomando notas durante la realización de las mismas, teniendo una duración promedia de 60 minutos a 1 hora 15 minutos. Para la realización de las entrevistas se administró un informe consentido y se emplearon pseudónimos en la investigación para proteger la identidad de todos los entrevistados. Todas las entrevistas fueron realizadas en una primera sesión salvo una, en la que el investigador entrevistó de nuevo a una participante para aclarar y profundizar en algunos aspectos considerados de interés para la investigación. Se practicó un estilo de entrevista activa incluyendo un guión de entrevista semiestructurado.

Tras la transcripción literal de las entrevistas toda la información fue transcrita y sometida a un primer análisis de discurso siguiendo las pautas marcadas desde la «grounded theory» (Strauss \& Corbin, 2002). El primer paso fue hacer visibles las teorías sustantivas de los participantes; para ello se realizó una codificación abierta que permitió emerger las primeras categorías. Desde la matriz de intersección de categorías de doble entrada obtenida con el programa NUDIST VIVO 10 se fue reconstruyendo el discurso de los personajes clave, para pasar a un
Tabla 2 .

Cruce de los discursos de los personajes clave relacionados con la perspectiva de género en el deporte español de competición.

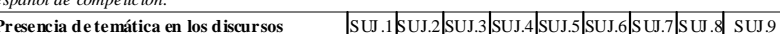
COMISIÓN DE MUJER Y DEPORTE

Visión positiva de la creación de la CMD del

CSD

Interés por la creación de más organismos

deportivos con perspectiva de género

Insuficiencia de las medidas tom adas por los

organismos con perspectiva de género

DISCRIMINACIÓ N POR RAZÓN DE

GÉNERO

Nula proyección deportiva profesional de

deportista en categ orías inferiores

Existencia de barreras que frenan la evolución

deportiva de las deport istas

Reconocimiento del mérito deportivo inferior

de los deportistas

Más exigen cia para ser reconocidas igual que

los deportistas

MARCO L EGISLATIVO

Valoración positiva de la entrd

Orgánica de Igualdad de 2007
Ont

Aplicación de la Ley Orgáni

Valoración positiva de la actual ley del Deporte

de 1990 en referencia al deporte femenino

Necesidad de la configuración de un nuevo

marco normativo en referencia al dep orte

Establecimiento de política de cuotas en las

Visión positiva del modus operandi de las

Inmovilidad de las federaciones en una unidad

estructural anticuada

Necesidad de la profesiona lización de la

Veportis

Tratami ento del asunto por parte de los

organismos deportivos españ oles

Necesidad del tratamiento de esta temática por

organismos competentes

MEDIOS DE COMUNICACIÓNY

LENGUAJE SEXISTA

Nula visib ilidad de la mujer deport ista en lo

medios de comunicación

Representación sexista de la im agen de la

deportista en los medios de comunicación

Lenguaje sexista en el deporte

Poco/muy poco reconocimiento de la deportista

por la sociedad es pañola

Positi vo avance de la deportista es pañola de alt

nivel en comparación con otros países

análisis comparativo de las percepciones de los participantes. Tal y como se puede ver en la tabla 2, se han recogido las principales temáticas aparecidas y se muestra (celda resaltada en gris) la presencia o ausencia de las mismas en las percepciones de cada uno de los participantes en esta fase de la investigación (ver tabla 2).

\section{Resultados}

Labor, funciones y valoración de los organismos con perspectiva de género en las federaciones deportivas españolas

La unanimidad de los entrevistados considera importante la creación y funciones de la Unidad de Mujer y Deporte creada bajo la orden Pre/525/2005, de 7 de marzo dentro del CSD, aunque según la opinión de algunos protagonistas (ver tabla 2) todavía existe cierto lastre en las federaciones deportivas españolas que no se suprimeúnicamente con la creación de más órganos específicos con perspectiva de género, sino con la mejora de la eficacia y eficiencia de los ya existentes. Algunos entrevistados opinan que los órganos con perspectiva de género en muchas ocasiones sólo sirven para cubrir el expediente y no atienden a una planificación adecuada a los objetivos a cumplir y una evaluación de la misma.

Sujeto 8 (ex-profesora universitaria):»El hecho de crear órganos no soluciona los problemas. Considero que algunos de los órganos que se han creado sólo sirven para «cubrir el expediente» y justificar que se hace algo. Me parece más importante y eficaz que se incluya a mujeres en la gestión y dirección de las federaciones deportivas y en los diferentes organismos e instituciones que dirigen el deporte y que se obligue a cumplir la Ley de Igualdad en el ámbito deportivo. En todo caso, si se crean comisiones específicas, éstas deberán desarrollar programas y evaluarlos para saber si su acción tiene repercusiones reales porque de lo contrario se convierten en órganos absolutamente ineficaces».

Existe unanimidad sobre la necesidad de apoyar medidas específi- 
cas y contundentes como la dotación a los órganos con perspectiva de género de las federaciones de autonomía directiva para tomar sus propias decisiones contando con la voluntad política expresa del personal federativo, inclusión de más mujeres en los puestos directivos de las federaciones, formación del personal federativo en perspectiva de género, planificación/evaluación deportiva de los programas de mujer y deporte y la aplicación estricta de la ley de igualdad en el ámbito deportivo.

\section{Discriminación por razón de género}

La mayor parte de los entrevistados reseñan que las mujeres deportistas tienen que hacer frente a barreras «sutiles» o «invisibles» ya sean de índole educativa, legislativa, cultural o social que frenan su práctica deportiva. Aunque la respuesta predominante es que actualmente no existen barreras visibles generales que impidan que las deportistas realicen actividad física de carácter competitivo sí que se encuentran con obstáculos difíciles de remoción a lo largo de su trayectoria deportiva. Culturalmente, ya desde inicios tempranos, son las familias las que estimulan a las niñas a elegir un itinerario deportivo que no entre en conflicto con los estereotipos tradicionales de género, dejando a un lado los deportes considerados tradicionalmente «masculinos».

Sujeto 7 (periodista deportiva): «En la actualidad siguen siendo unas barreras invisibles porque no es que tenga una barrera que por ser niña no pueda practicar uno u otro deporte. Por ejemplo, por decirte algún deporte, normalmente por esta cadencia que tienen padres y madres, los niños quieren apuntarlos a fútbol o a deportes un poco más agresivos y a las niñas las seguimos apuntando a gimnasia, patinaje».

Tal y como se desprende del discurso de los entrevistados, ni los medios de comunicación, ni la legislación deportiva vigente ampara una proyección profesional de la deportista en el deporte de competición, no existiendo referentes de mujeres deportistas para las niñas o adolescentes en la sociedad que las animen a dedicarse profesionalmente al deporte. De hecho, según una de las entrevistadas existen además lagunas en la legislación deportiva vigente que no contemplan un desarrollo profesional de la deportista.

Sujeto 6(abogada deportiva):»Creo que hace falta en nuestro país un diseño de la carrera profesional de la deportista, cuando esa mujer se presenta o se plantea dedicarse profesionalmente a esa actividad deportiva, ahí empieza el problema, en el Real Decreto 1006/1985, de 26 de junio, por el que se regula la relación laboral de los deportistas profesionales para mí hay elementos que no contempla y que debería contemplar, un elemento por ejemplo sustancial, el tema del embarazo, el tema de la lactancia, el tema de la maternidad, no lo contempla ese real decreto, yo tendría que irme al estatuto de los trabajadores evidentemente, pero yo puedo afirmar hoy desde mi experiencia profesional que hay deportistas que son despedidas cuando se quedan embarazadas, eso en el derecho laboral común no se puede admitir es una aberración jurídica ¿cuál es otro elemento para mí importante?, el tema de la retribución, vale, hay muchas deportistas que dedican gran parte de su tiempo a esa actividad que podríamos definir denominar ya profesional y tienen una retribución no como deportista, no comojugadora de fútbol, se le hace un contrato que puede ser una panadería, empleada de hogar y demás para compensar esa falta de retribución económica o el hecho de cotizar por ello en la seguridad social, esa situación se está dando ahora».

Además, en las deportistas que son profesionales existe entre ellas una inferioridad interiorizada respecto a su papel como profesional del deporte que no ocurre en los hombres profesionales:

Sujeto 6(abogada deportiva):»Yo te reconozco que hayjugadoras, deportistas, que van, cuando van a mi despacho para hacerme consultas, para que les lleve algún asunto y al mismo tiempo me van chicos, el chico va de profesional y me pide que como profesional que actúe y denuncie, las chicas van con miedo, no se cree que sea profesional»

La respuesta predominante es que al igual que ocurre en todos los ámbitos de la sociedad, las mujeres tienen que demostrar su valía mucho más que el hombre para ser igualmente reconocidas. Así mismo, en igualdad de condiciones las deportistas se encuentran en segundo plano en cuanto a todo el engranaje del sistema de competición: peores horarios, distribución desigual de recursos materiales, humanos y económicos. Respecto a la cuantía de los premios, los entrevistados reconocen que aunque se han incrementado las ayudas concedidas por las administraciones públicas y las becas de ayuda al deporte olímpico (ADO) son igualitarias entre sexos, considerándolas satisfactorias; todavía es muy precario el patrocinio deportivo privado que se presta a las deportistas y el dinero destinado a la promoción deportiva desde las entidades privadas

\section{Marco legislativo en materia deportiva}

En referencia al marco normativo en el deporte, muchos de los entrevistados consideran que debe hacerse un ejercicio de reflexión y adaptar la legislación deportiva vigente a la estructura del deporte actual que ha variado considerablemente desde la aplicación de la ley 10/1990 de 15 de Octubre. Más específicamente, y en el caso de la igualdad de género, el marco normativo en materia deportiva debe adaptarse a los requerimientos específicos de las deportistas, ya que no se reconocen aspectos fundamentales y trascendentales en la trayectoria deportiva de las deportistas como el embarazo, lactancia, maternidad, conciliación y retribución. En este sentido varios de los entrevistados, apuntan que debe ponerse en marcha una ley del deporte profesional que recoja los aspectos mencionados anteriormente para llevar a cabo una remoción de aspectos de discriminación que se están produciendo y que vulneran derechos fundamentales de las deportistas.

Sujeto 6 (abogada deportiva):»Pongo sobre la mesa varias cuestiones, la primera, un elemento diferenciador, el tema del embarazo, el tema de la lactancia, el tema de la maternidad, esto tal como está a hora regulada yo afirmo que hay jugadoras que hay deportistas que se les extingue, se les rescinde el contrato cuando se quedan embarazadas y que no puede admitirse y la otra cuestión importante es el tema de las retribuciones, otro tema paradójico por ejemplo que se da en el deporte y que no se da en cualquier otra esfera del ámbito laboral».

Incluso muchas deportistas a pesar de dedicarse profesionalmente a su actividad deportiva, no tienen un contrato profesional con sus equipos, clubes que las avale como profesionales del deporte, por lo que no tienen los derechos laborales como trabajadora o tienen contratos que no se corresponden con su dedicación real.

Sujeto 6 (abogada deportiva):»Un ejemplo muy sangrante, que una mujer sé que está practicando deporte, una triatleta o cual sea, tiene una lesión grave, una lesión de tal magnitud que le impide, que la incapacita laboralmente para ejercer cualquier actividad profesional, como esa mujer no han cotizado por ella, como su profesión real, ¿en qué situación se encuentra?, eso se puede producir perfectamente en este país y está en la absoluta precariedad, eso hay que, eso son cuestiones que tenemos que subsanar, en los tiempos actuales, eso se da, eso se da en la sociedad actual, no, no se puede permitir».

La respuesta predominante es que la Ley Orgánica 3/2007, de 22 de marzo para la igualdad efectiva de mujeres y hombres ha supuesto un paso muy importante en el reconocimiento y defensa de los derechos de las mujeres.

Sujeto 6 (abogada deportiva):»La Ley de Igualdad es importante, la Ley de Igualdad establece un artículo específico, el 9, sobre el tema del deporte, pero muy en general, pero creo que era necesaria, creo que es una ley muy importante y yo como abogada y como jurista para mí está siendo un instrumento capital a la hora de en el juzgado y a la hora de, podemos decir, pelear estos temas en órganos de la creación pública o distintas instancias deportivas es un referente que se trata una ley orgánica que evidentemente está por encima de la ley del deporte que este tipo de normativa de rango inferior, me parece que era necesaria».

No obstante, consideran que la ley tiene ciertas lagunas en lo referente a la estructura del deporte, no produciéndose una aplicación exhaustiva de la ley y no teniendo el personal directivo de muchas federaciones la preparación necesaria para su aplicación debido a la poca formación en perspectiva de género y al modus operandi de muchas federaciones que lastran modos de hacer viciados como consecuencias 
del lastre o inercia del pasado difíciles de desgajar.

Muchos participantes se posicionan a favor de la política de cuotas en las federaciones con el consecuente cambio del Real Decreto 1026/ 2007 de 20 de Julio para romper la inercia de las federaciones e incluir más mujeres en sus filas. La atribución a esta problemática muchos de los entrevistados la atañen a los horarios de muchas federaciones que no permiten la conciliación personal, familiar y laboral de la mujer, y a que muchos de los cargos son voluntarios no retribuidos y muchas mujeres no se sienten válidas en las federaciones debido a su tradicional funcionamiento que las coartan y acaban desistiendo de sus funciones.

Sujeto 1(gestora deportiva de la Comunidad Autónoma):»Hay algunas federaciones que con los planes de igualdad actuaban incluso con cierta agresividad, ¿es que discriminamos a las chicas? no para nada. El implantar un plan de igualdad en una empresa o asociación no quiere decir que discrimines, lo que vas es a mejorar la situación de igualdad tanto para hombres como para mujeres. Son temas formativos simplemente en materia de género. $Y$ eso muestra poco interés, son reacios en cierto sentido».

\section{Violencia machista}

Existe consenso en la práctica totalidad de los entrevistados en que es importante que se aborde este asunto en España, pero desde una perspectiva que no afecte al deporte de una manera peyorativa, y se levante una alarma social que creen innecesaria. Hay algunos entrevistados que creen que el tema de la violencia de género en el deporte es un tema tabú y que no se quiere investigar.

Establecen que el deporte no es ajeno a esta realidad ya que las relaciones de poder incurren en problemas de diversa índole relacionados con esta temática y que se tiene que llevar a cabo una campaña de concienciación de todos los agentes involucrados en el deporte, concienciando a las deportistas que denuncien sin miedo a que se puedan tomar represalias contra ellas y que tengan que abandonar su práctica deportiva.

\section{Medios de comunicación y lenguaje sexista}

La respuesta predominante de los protagonistas es que la presencia de la deportista en los medios de comunicación es prácticamente nula y muchas veces acentuando los estereotipos sociales de género, recreando de esta manera una imagen erotizada y sexuada de la mujer deportista no publicitando sus logros deportivos.

Sujeto 4 (gestora federativa):»Yo diría, más bien, el trato que no reciben porque prácticamente no están presentes. No obstante, cuando se las incluye, suele ser ratificando los estereotipos sociales de género. Incluso, destacándolos y ridiculizándolos».

Los entrevistados afirman que la deportista española es poco reconocida en la sociedad porque no se publicita su imagen, sólo son reconocidas las llamadas «deportistas mediáticas», aquellas que destacan muchísimo por sus logros deportivos o porque su deporte no rompe con los estereotipos de género.

Sujeto 7 (periodista deportiva):»Hay unas pocas que están muy bien reconocidas porque están, voy a llamarlas entre comillas «mediáticas», la campeona del mundo de natación sincronizada, pero porqué, porque cumple unas normas que todos se han molestado en señalar la sirena de las piscina, y la idea de esta deportista en la piscina como deportista y como campeona que es con más de 20 medallas, en competiciones a nivel internacional, eso nadie lo puede negar, pero ha tenido una especie de relevancia ya no sólo por sus medallas sino por el deporte que practicaba». En cuanto al lenguaje la totalidad de los entrevistados creen que se sigue utilizando un lenguaje no inclusivo que invisibiliza a las mujeres y muchas veces las trata de manera despectiva.

\section{Discusión}

Los resultados de la investigación ponen de manifiesto la importancia de las políticas deportivas con perspectiva de género para romper las barreras visibles o sutiles que impiden la equidad de género en el deporte (Blakemore \& Drake, 1996). Además, según la última Conferencia del
COI sobre Mujer y Deporte «La Declaración de los Ángeles» desde que se aplican plenamente los programas y procesos para ayudar a las mujeres a acceder de forma sistemática a los niveles más altos de la administración deportiva y la competición, el número de participantes femeninas en el deporte ha crecido de manera exponencial. Sin embargo, Shaw \& Penney (2003) y Puig \& Soler (2003) ponen de manifiesto la problemática inherente de incluir las políticas deportivas con perspectiva de género en las organizaciones deportivas. Tal y como se desprende el discurso de los entrevistados existe una reticencia por parte de los organismos deportivos de comprometerse formalmente con la realización de medidas específicas y contundentes como la dotación a las comisiones de mujer y deporte de las federaciones de autonomía directiva para tomar sus propias decisiones o de la mejora de las ya existentes (Hoeber \& Frisby, 2001).Este resultado es similar al estudio realizado por Shaw \& Penney (2003) en los organismos deportivos ingleses donde la autora y el autor realizan una crítica sobre la implantación y seguimiento de las políticas deportivas con perspectiva de género. Respecto a las ayudas concedidas por las administraciones públicas y las becas ADO los entrevistados las consideran satisfactorias; aunque todavía es muy precario el patrocinio deportivo privado que se presta a las deportistas y el dinero destinado a la promoción deportiva desde las entidades privadas. Este resultado coincide con el estudio de (Cabello et al., 2011) sobre el modelo de análisis del deporte español de siglo XXI en el que existe unanimidad de los dirigentes federativos sobre las bondades del Plan ADO como modelo impactante siempre mencionado cuando se refiere a la captación de fondos privados, no obstante barajan cierta incertidumbre sobre si esta propuesta sería replicable en otros contextos del deporte: planes de promoción, competiciones de alto nivel al margen de los Juegos Olímpicos y las ayudas concedidas por las administraciones públicas. La mayoría de los entrevistados consideran insuficiente el número de mujeres en puestos de responsabilidad en los organismos deportivos como muestran estudios realizados hasta la fecha (Alfaro et al., 2012; Peyró, 2003; Pfister, 2006; Robles \& Escobar, 2007).

Las mujeres en las organizaciones deportivas españolas siguen sin alcanzar la cuota del 20\% propuesta para el año 2005 en la Conferencia de París por el Comité Olímpico Internacional. Tan sólo el CSD ha incorporado más mujeres a sus filas alcanzando este año 2014 el35,71\% de representación femenina en su organismo. No obstante, se trata de un caso aislado ya que el grueso de mujeres en las federaciones deportivas pertenece al comité de árbitros y jueces (24\%), estando las mujeres infrarrepresentadas en las Presidencias de las Federaciones (4,5\%), Asambleas Generales (11\%) Comisiones Delegadas (10,6\%) y Juntas Directivas (12,3\%). La segregación de género en el mercado laboral en España se caracteriza por ser tanto vertical como horizontal, encontrándose las mujeres tal y como afirma Pfister (2006) en los niveles inferiores de la jerarquía de las organizaciones y con menores oportunidades de promoción a favor del sexo masculino (Davidson \& Black, 2001). A nivel internacional la situación no es diferente ya que en la última Conferencia del COI sobre Mujer y Deporte se ha puesto de manifiesto que a pesar de que se han invertido recursos para situar a mujeres en puestos de liderazgo en las organizaciones deportivas el número de mujeres electas no ha aumentado al mismo ritmo que su participación como deportistas, por lo que se insta a los comités olímpicos nacionales, federaciones internacionales y las federaciones nacionales a que en los ciclos electorales en el futuro, se logre una representación más equitativa en sus Comités Ejecutivos.

La preocupación de los entrevistados por la violencia machista y acoso y abuso sexual en el deporte de competición se ha visto refrendada por numerosos estudios a nivel internacional (Brackenridge, 2001; Svela Sand et al., 2011; Vázquez et al., 2002). Este año 2004, de acuerdo con la moción aprobada por el senado en 2013 en la que se instaba al gobierno a la adopción de determinadas medidas para evitar el abuso sexual e infantil en el deporte, el CSD ha desarrollado el «Plan de detección, actuación, prevención de los abusos sexuales en el mundo del deporte», siendo ya algunas federaciones las que se han adherido como la Federación Española de Piragüismo. No obstante, el CSD insta al 
resto de federaciones para su adhesión en la temporada deportiva 2014/ 2015.

Según se recoge en la reciente publicación del CSD (López, 2011) los medios de comunicación ejercen una enorme influencia en el conocimiento de la realidad social, ayudando a conformar la opinión pública y a la adopción de conductas personales y sociales. Los resultados del estudio ponen de manifiesto que el deporte femenino de competición se encuentra al margen en cuanto a su presencia y visibilidad de sus logros deportivos en los medios de comunicación (Élida et al., 2012, Crolley y Tesso; 2007; Latorre et al., 2007; López, 2011; Sainz de Baranda, 2013). Del mismo modo, los resultados del estudio de (Bissell \& Duke, 2007) mostraron que la sexualidad de la mujer era utilizada para promocionar a la deportista y/o el deporte practicado. Así mismo, el lenguaje en la mayoría de las ocasiones es un lenguaje sexista que redunda en la imagen estereotipada de la deportista (Élida et al., 2012; Jones, 2011). Las referencias normativas actuales en cuanto a la aplicación de un lenguaje no sexista se encuentran en la Ley Orgánica 3/2007 para la igualdad efectiva entre mujeres y hombres, cuyo art. 14 estipula que serán criterios generales de actuación de los poderes públicos, «la implantación de un lenguaje no sexista en el ámbito administrativo y su fomento en la totalidad de las relaciones sociales, culturales y artísticas». En el Consejo Superior de Deportes, desde la Unidad del CSD y través del libro «Hablamos de Deporte» (Alfaro, Bengoechea \& Vázquez, 2010) las autoras hacen un análisis exhaustivo sobre el uso del lenguaje sexista en el ámbito deportivo en todos los niveles. También académicos en Estados Unidos han abogado por el empleo de un lenguaje no sexista en los programas de gestión deportiva (Parks \& Roberton, 2002).

\section{Conclusiones: reconceptualización del modelo existente}

A la vista de los resultados el modelo deportivo español precisa de un amplio debate y reflexión que ponga en cuestión muchos de los elementos esenciales que tienen que ver con el desarrollo de la deportista en el deporte español de competición. Se hace necesario un cambio en la legislación y en el modo de gestionar de los entes deportivos que permita a la deportista española competir en igualdad de condiciones que los deportistas españoles.

Partiendo de la legislación deportiva, es prioritario que se recojan aspectos esenciales y específicos de la deportista que condicionan su trayectoria deportiva (maternidad, conciliación, retribución...) y que se regule su profesionalización para que pueda dedicarse al deporte de competición como a cualquier otra profesión. En referencia a la legislación española con perspectiva de género es necesario que se cumpla en todas sus vertientes y especialmente en el ámbito deportivo y por parte de todos los organismos implicados en el deporte. Las federaciones deportivas españolas deben impulsar sus políticas de género en el seno federativo incorporando más mujeres a sus puestos de toma de decisión, formando al personal federativo en una visión con perspectiva de género y llevando a cabo programas de acción con el objetivo de incrementar el número mujeres en todos los estamentos federativos adaptándose a la demanda existente. Teniendo en cuenta la invisibilidad de la deportista española en los medios de comunicación es necesario que se creen sinergias comunes entre los medios de comunicación y los entes deportivos para visibilizar los logros deportivos de la mujer deportista libre de estereotipos de género y con un lenguaje inclusivo que sirva de modelo de referencia para las niñas y mujeres de la sociedad española. En definitiva sería conveniente la reconceptualización del modelo, en el que se tenga en cuenta en el modelo deportivo actual un cambio en la legislación deportiva con el objetivo de mejorar la situación de la deportista española, y una mejora de las políticas de género en materia deportiva enfocada a las federaciones deportivas españolas con el objetivo de mejorar el sistema federativo y como consecuencia el deporte femenino español de competición.

\section{Referencias}

Alfaro, E., Vázquez, B., Gallardo, J., \& Ferro, S. (2012). Mujeres en puestos de responsabilidad dentro de las organizaciones públicas deportivas de la Comunidad de Madrid. Ágora para la Educación Física y el Deporte, 15, 40-53.
Alfaro, E., Bengoechea, M. \& Vázquez, B. (2010). Hablamos de deporte: en femenino y en masculino. Madrid: Instituto de la Mujer (Ministerio de Sanidad, Política Social e Igualdad).

Brackenridge, C.H., Spoilsports: Understanding and Preventing Sexual Exploitation in Sports, Routedge, London, 2001.

Brakemore, K. \& Drake, R. (1996). Understanding equal opportunity policies. London: Prentice Hall.

Bertalanffy, L. V.(1968). General System Theory. Foundations, Development,Applications. New York: George Braziller.

Bissell, K. L, \& Duke, A. M. (2007). Bump, set, spike: An analysis of commentary and camera angles of women's beach volleyball during the 2004 summer olympics. Journal of Promotion Management, 13 (1-2), 35-53.

Bryman, A. (2004). Social research methods. New York: Oxford University Press.

Cabello, D., Rivera, E., Trigueros, C. \& Pérez, I. (2011). Análisis del modelo del deporte federado español del siglo XXI. Revista Internacional de Medicina y Ciencias de la Actividad Física y el Deporte, 11(44), 690-707.

Consejo Superior de Deportes. (2013). Anuario de estadísticas deportivas de 2013. Ministerio de Educación Cultura y Deporte.

Crolley L. \& Tesso, E. (2007). Gendered narratives in Spain: the representation of female athletes in Marca and el País. International Review for the Sociology of Sport, 42(2), 149-166.

Davidson, P. \& Black, R. (2001). Women working in male roles: A New South Wales and Victorian case study. Parks and Leisure Australia, 4(4), 10-15.

Fink, A. (1995). How to sample in surveys. Thousand Oaks: Sage.

Fraile, A. \& Vizcarra, T. (2009). La investigación naturalista e interpretativa desde la actividad física y el deporte. Revista de Psicodidáctica, 14(1), 119-132.

García Ferrando, M. (1987) La mujer en el deporte de alta competición: conflicto de roles y adaptación al modelo deportivo dominante. El caso del atletismo español, en publicaciones de Mujer y Deporte, Madrid: Ministerio de Cultura. Instituto de la Mujer, 21-51.

García Ferrando, M. (2002). La encuesta. En F. Alvira, M. García Ferrando, y J. Ibáñez (comps.), El análisis de la realidad social. Métodos y técnicas de investigación (pp. 167-202). Madrid: Alianza Editorial.

González Tirados, G (2010). El problema de género en las estructuras empresariales españolas del siglo XXI. Mujeres y Economía. Instituto De Ciencias De La Educación, 852, pp. 113-125.

Hartmann-Tens, I. \& Pfister, G. (2003). Sport and women, social issues in international perspective. International Society for Corporative Physical Education and Sport.

Hoeber, L. \& Frisby, W. (2001). Gender equity for athletes: Rewriting the narrative for this organizational value. European Sport Management Quarterly, 1, 179-209.

Jones, A. H. (2011). Visual and verbal gender cues in the televised coverage of the 2010 winter olympics. International Journal of Interdisciplinary Social Sciences, 6(2), 199-216.

Latorre Román, P. y otros. (2007). Mujer, deporte y medios de comunicación. Revista digital de Buenos Aires, 11(106).

López, P. (2011). Deporte y Mujeres en los medios de comunicación. Recomendaciones y sugerencias. Consejo Superior de Deportes, Madrid.

Manrique Arribas, J.C. (2003). La educación física femenina y el ideal de mujer en la etapa franquista. Revista Internacional de Medicina y Ciencias de la Actividad Física y el Deporte, 10, 83-100.

ManriqueArribas, J.C., López, V.M., Torrega, L.M. \& Mangos, R. (2008). La labor formativa desarrollada por la sección femenina de la falange en la preparación de sus mandos e instructoras durante el periodo franquista. Historia de la Educación, 27, 347365

Parks, J. B. \& Roberton, M. A. (2002). The gender gap in student attitudes toward sexist/ non sexist language: Implications for Sport Management education. Journal of Sport Management, 16(3), 190-209.

Peyró Santana (2002). Las Mujeres en la Dirección, Gestión y Organización de la alta competición Deportiva. En VV.AA (Eds) Las mujeres en la alta competición deportiva (Madrid Consejo Superior de Deportes), 129-148.

Pfister, G. (2010). Women in Sport-gender relations and futures perspectives, Sport in Society, 13(2), 234-248.

Puig, N. \& Soler, S. (2003). Women and sport in Spain. En G Pfister \& I. Hartmann-tews (Eds.), Women and sport: a crosscultural analysis (pp. 83-101). London: Routledge.

Puig., N. \& Soler, S. (2004). Mujer y deporte en España: Estado de la cuestión y propuesta interpretativa, Apunts: Educación Física y Deportes, 76, 71-78.

Robles., F. \& Escobar, K. (2007). Mujeres en los órganos de gobierno de las organizaciones deportivas españolas 2002-2006.Comité Olímpico Español.

Sainz de Baranda, C. (2013). Mujeres y deporte en los medios de comunicación. Estudio de la prensa española. (Tesis doctoral). Universidad Carlos III de Madrid.

Shaw, S. \& Penney, D. (2003). Gender equity policies in national governing bodies: An oxymoron or a vehicle for change? European Sport Management Quarterly, 3, 78102.

Strauss, A. \& Corbin, J. (2002) Bases de la investigación cualitativa. Técnicas y procedimientos para desarrollar la teoría fundamentada. Colombia: Universidad de Antioquia.

Svela Sand, T., Fasting, K., Chroni, S. \& Krorre, A. (2011). Any Consequences for the Prevalence of Sexual Harassment?, International Journal of Sports Science \& Coaching, 6, 229-241.

Torres García, D. (2005). Propuesta de programación didáctica coeducativa en educación física en educación primaria: un caso de la sierra de Madrid, Retos. Nuevas Tendencias en Educación Física, Deporte y Recreación, 8, $43-49$.

Vazquez, B.; Bastanchuri., E. \& Fernández Heyden, N. (2002). El acoso sexual en el deporte de competición. Las mujeres en la alta competición deportiva. Madrid: Ministerio de Educación Cultura y Deporte. Consejo Superior de Deportes, 87104. 